Research Article

\title{
Optimization Analysis of Smart Steel-Plastic Geogrid Support for Tunnel
}

\author{
Qin Liu, ${ }^{1}$ Jiankun Guo, ${ }^{1}$ Lei Liu, ${ }^{1}$ Kunpeng Huang, ${ }^{1}$ Wei Tian, ${ }^{1}$ and Xinzhi Li $\mathbb{D}^{2}$ \\ ${ }^{1}$ School of Civil Engineering, Chang'an University, Xi'an 710064, China \\ ${ }^{2}$ State Key Laboratory of Mechanical Behavior and System Safety of Traffic Engineering Structures, \\ Shijiazhuang Tiedao University, Shijiazhuang 050043, China \\ Correspondence should be addressed to Xinzhi Li; hshaocun@163.com
}

Received 16 October 2020; Revised 18 November 2020; Accepted 10 December 2020; Published 29 December 2020

Academic Editor: Chunshun Zhang

Copyright (c) 2020 Qin Liu et al. This is an open access article distributed under the Creative Commons Attribution License, which permits unrestricted use, distribution, and reproduction in any medium, provided the original work is properly cited.

\begin{abstract}
With the concept of smart geogrid coming out, many scholars have built optical fiber into the geogrid to form a kind of smart geogrid material with self-sensing function of structural deformation. It can not only reinforce the parts with potential safety hazards, but also have the functions of safety monitoring, intelligent prevention, and control of engineering disasters, which is of great significance for ensuring the safety of tunnel construction and improving the tunnel monitoring methods. Based on predecessors' research on smart geogrid tensile calibration experiment and sensor method simulation and experimental verification, this paper analyzes the smart geogrid and the tunnel surrounding rock as a whole, to study the deformation coordination mechanism between the geogrid material and the tunnel surrounding rock. Referring to the relevant engineering practice case, through finite element numerical simulation, the optimal layout of smart geogrid material was explored, and the principle of discrete curvature reconstruction curve sensing of smart geogrid was optimized by simulating the working conditions of different construction methods and supporting conditions, in order to provide a theoretical basis for the application of smart geogrid material in practical tunnel engineering.
\end{abstract}

\section{Introduction}

Tunnel deformation monitoring has always been a common problem in engineering, especially for the project with high ground stress, soft surrounding rock, penetrating cracks, and other special engineering geology, and the precision and means of monitoring has become a major problem. Fiber Bragg grating (FBG) sensing technology has the characteristics of strong anti-interference ability, high sensitivity, easy to be embedded, and attached to the surface of the structure and can realize long-distance transmission. It has become a popular intelligent monitoring method in tunnel engineering [1]. By embedding FBG into flexible substrate (such as steelplastic geogrid) with high flexibility, high ductility, and free bending characteristics, a flexible sensor for measuring morphology, displacement, strain, and other variables is formed [2-3], which will be a trend in the future development of sensors in civil engineering field.
Since the fiber Bragg grating sensors which were first used in concrete structure achieved good results [4], they have been extended to the monitoring of infrastructure, dam, traffic, tunnel, slope, and other complex systems, for example, lateral deformation monitoring of embankment soft soil [5]; monitoring of bridge prestress loss [6, 7]; pressure monitoring of tunnel's surrounding rock and monitoring of rock bolt support quality [8,9]; internal deformation monitoring of landslide [10]; and monitoring deformation of a pit-in-pit foundation [11].

With the birth and development of flexible sensors, at first, some scholars proposed to stick the resistance strain gauge to the surface of steel-plastic geogrid for surface strain monitoring [12]. However, the measurement results of the strain gauges are susceptible to interference, and they are not placed inside the material, which does not form a real smart material. In recent years, people have embedded FBGs into flexible structures such as silicone rubber sheets and synthetic silicone resins and realized the reconstruction of the 
three-dimensional shape of the object surface through the principle of discrete curved reconstruction curve $[13,14]$. This kind of flexible sensor has been applied in biomedical and aviation fields. In 2016, the built-in sensor smart geogrid (SEGG) came out, and its response to tensile strain in the soil was analyzed in numerous studies $[15,16]$. The results showed that this technology is expected to replace the traditional geotechnical structure performance monitoring instrument. In China, scholars of Shandong University developed a smart geogrid with a built-in fiber grating sensor which has integrated measurement and reinforcement function, and through tensile calibration experiment, model experiment, and numerical simulation, the consistency of fiber grating strain and geogrid strain and the feasibility of smart geogrid sensing principle are analyzed [17, 18]. Scholars of China University of Mining and Technology had developed a metallized encapsulated FBG smart geogrid for mining. The curvature simulation test of its components is also carried out to verify the relationship between wavelength conversion and strain of FBG under stress deformation [19].

However, most of the existing research is based on the flexible sensor (smart geogrid) as an individual to analyze the applicability of its sensing principle. In practical engineering, geogrid is inseparable from surrounding rock, primary support, secondary lining, and other support methods. Therefore, it is of great importance to study the deformation coordination mechanism between geogrid material and surrounding rock as a whole. In this paper, through the PLAXIS 3D finite element simulation, the feasibility of the smart geogrid discrete curvature reconstruction curve sensing principle under actual working conditions is analyzed under the coupling of different construction methods, supporting conditions, and other factors, and an optimized design is proposed for it. We explore the optimal layout of smart geogrid materials in actual projects.

\section{Smart Geogrid Displacement Response Mechanism}

2.1. Principle of Smart Geogrid Curvature Sensing. As a common network structure, geogrid can be regarded as a collection of multiple spatial curves. Assuming that each curve is composed of several arcs, when the curvature and arc length of each arc are determined, the reconstruction of the curve can be realized, thus extending to the deformation network of the geogrid.

When the external physical environment changes (mainly temperature and strain), the wavelength of fiber Bragg grating will change accordingly. Therefore, the temperature and strain change rules can be reflected according to the change of the central wavelength of fiber Bragg grating. Assuming that each curve is arranged with a series of FBG fiber grating sensors in the longitudinal direction, the relationship among the arc curvature, arc length, strain, and wavelength change is established through the relationship diagram of the smart grid curvature sensing principle in Figure 1 [20].

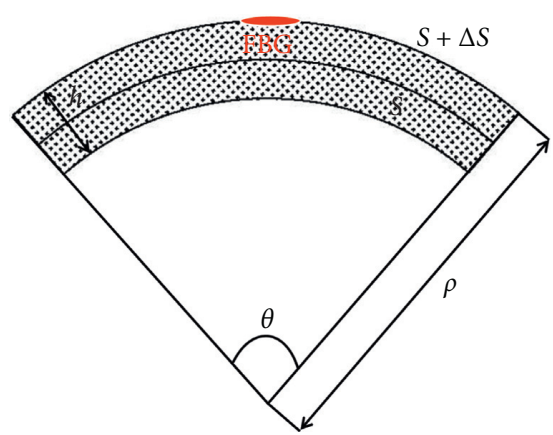

FIGURE 1: Principle of smart geogrid curvature sensing.

Assuming that FBG and geogrid have formed a good whole, the strain measured by FBG is the geogrid strain here (the following strain $\varepsilon$ is the geogrid strain value). When the curve element is bent, the length of the neutral layer is still the arc length $s$, the arc length from the neutral layer $h / 2$ is $s+\Delta s, \rho$ is the radius of curvature of the arc, and $\theta$ is the center of the arc angle. According to the principles of material mechanics:

$$
\frac{s}{\rho}=\frac{s+\Delta s}{\rho+h / 2}=\theta .
$$

Without considering the change of temperature factor, the central wavelength of FBG is only affected by strain. Equation (1) can be simplified as

$$
k=\frac{1}{\rho}=\frac{2 \cdot \Delta s / s}{h}=\frac{2 \cdot \varepsilon}{h},
$$

where $\varepsilon$ is the strain value at the neutral layer of the geogrid. According to the principle of FBG sensor, the central wavelength variation of the fiber grating is

$$
\Delta \lambda=\lambda\left(1-P_{e}\right) \cdot \varepsilon=K_{e} \cdot \varepsilon .
$$

Then, the relationship between the change in center wavelength and the curvature of this arc is

$$
k=\frac{2 \cdot \Delta \lambda}{h \cdot k_{\mathrm{e}}},
$$

where $k_{\mathrm{e}}$ is the sensitivity of FBG sensor, which is an inherent property of fiber grating material. Equation (4) shows that the curvature of circular arc is linear with the variation of central wavelength. The curvature value can be obtained by measuring the variation of central wavelength by FBG sensor, and it can also be obtained by substituting the strain value of geogrid according to formula (2).

\subsection{Principle of Curve Reconstruction with Discrete Curvature.} The commonly used methods of discrete curvature reconstruction include the recursive method, quadratic integral method, bilinear interpolation method, etc. [21] Considering the practical value of geogrid, this paper uses the recursive method to elaborate and further optimize its theory. When the distance between two points on the curve is close enough, the curve can be regarded as a microarc; it can be 
approximated by dividing the curve into several circular arcs. The curvature of end point of each arc can be linearly interpolated through the curvature of the start point and end point. Finally, according to the coordinates and curvature of the discrete points, the coordinates of each interpolation point can be deduced to realize the fitting and reconstruction of the curve [22].

As shown in Figure 2, the curve is divided into $\operatorname{arc} a_{0} a_{1}$, arc $a_{1} a_{2}$, arc $a_{2} a_{3} \ldots$. The starting coordinates of the microarc are $a_{0}\left(x_{0}, z_{0}\right)$, the ending coordinates are $a_{1}\left(x_{1}, z_{1}\right)$, the length of $\operatorname{arc} a_{0} a_{1}$ is $s_{1}$, and $s_{1}$ can be taken as the distance between two adjacent FBG sensors in the geogrid. $k_{0}$ is the curvature of the starting point $a_{0}, r_{1}$ is the radius of the microarc $a_{0} a_{1}$, and $r_{1}$ can be obtained by the following formula:

$$
r_{1}=\frac{1}{\overline{k_{1}}}=\frac{2}{k_{0}+k_{1}} .
$$

The end point coordinates $a_{1}\left(x_{1}, z_{1}\right)$ of the microarc $a_{0} a_{1}$ can be calculated by the following formula:

$$
\left\{\begin{array}{l}
\theta_{1}=s_{1} \cdot \overline{k_{1}}, \\
x\left(o_{1}\right)=0 \\
z\left(o_{1}\right)=\frac{1}{\overline{k_{1}}} \\
x_{1}=x\left(o_{1}\right)+\frac{\sin \theta_{1}}{\overline{k_{1}}}, \\
z_{1}=z\left(o_{1}\right)-\frac{\cos \theta_{1}}{\overline{k_{1}}} .
\end{array}\right.
$$

As shown in Figure 2, the length of micro-arc $a_{1} a_{2}$ is $s_{2}$, the point $o_{2}$ is the center of the microarc $a_{1} a_{2}, r_{2}$ is the radius of the microarc $a_{1} a_{2}, r_{2}$ can be obtained recursively according to formula (5). $\theta_{2}$ is equal to $\theta_{1}$ plus $\Delta \theta ; \Delta \theta$ is the central angle corresponding to the microarcs $a_{1} a_{2}$. According to Formula 6 recursively to Formula 7, the coordinates of $a_{2}\left(x_{2}, z_{2}\right)$ can be obtained by the mathematical relationship of the following formula:

$$
\left\{\begin{array}{l}
\theta_{2}=\theta_{1}+s_{2} \cdot \overline{k_{2}}, \\
x\left(o_{2}\right)=x\left(o_{1}\right)-\frac{\left(\overline{k_{1}}-\overline{k_{2}}\right) \cdot \sin \theta_{1}}{\overline{k_{1}} \cdot \overline{k_{2}}}, \\
z\left(o_{2}\right)=z\left(o_{1}\right)+\frac{\left(\overline{k_{1}}-\overline{k_{2}}\right) \cdot \cos \theta_{1}}{\overline{k_{1}} \cdot \overline{k_{2}}}, \\
x_{2}=x\left(o_{2}\right)+\frac{\sin \theta_{2}}{\overline{k_{2}}}, \\
z_{2}=z\left(o_{2}\right)-\frac{\cos \theta_{2}}{\overline{k_{2}}} .
\end{array}\right.
$$

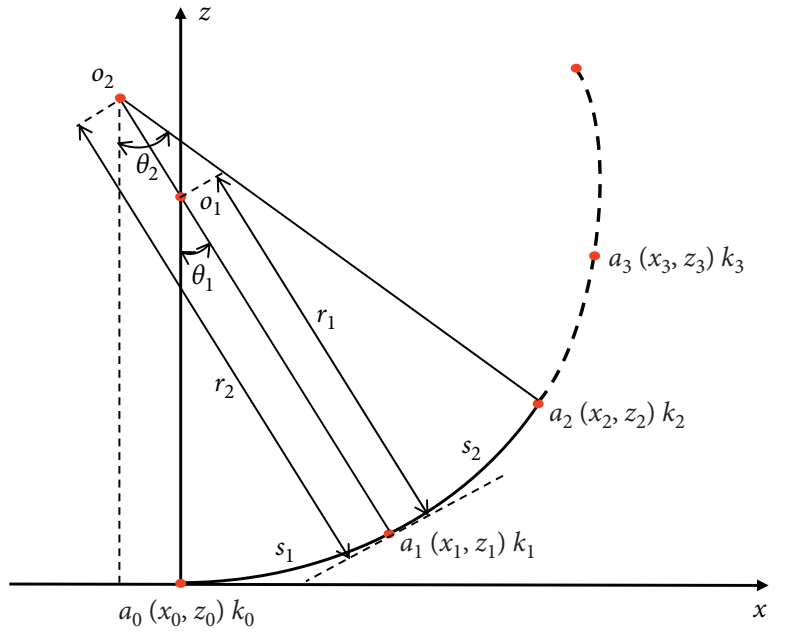

Figure 2: Discrete curvature reconstruction curve based on recursion method.

From Formulas 6 and 7, we can see that for any microarc $a_{\mathrm{n}} a_{\mathrm{n}+1}$, its coordinates $a_{\mathrm{n}}\left(x_{\mathrm{n}}, z_{\mathrm{n}}\right)$ and $a_{\mathrm{n}+1}\left(x_{\mathrm{n}+1}, z_{\mathrm{n}+1}\right)$ satisfy the following mathematical relationship:

$$
\left\{\begin{array}{l}
\theta_{n+1}=\theta_{n}+s_{n+1} \cdot \overline{k_{n+1}}, \\
x\left(o_{n+1}\right)=x\left(o_{n}\right)-\frac{\left(\overline{k_{n}}-\overline{k_{n+1}}\right) \cdot \sin \theta_{n}}{\overline{k_{n}} \cdot \overline{k_{n+1}}}, \\
z\left(o_{n+1}\right)=z\left(o_{n}\right)+\frac{\left(\overline{k_{n}}-\overline{k_{n+1}}\right) \cdot \cos \theta_{n}}{\overline{k_{n}} \cdot \overline{k_{n+1}}}, \\
x_{n+1}=x\left(o_{n+1}\right)+\frac{\sin \theta_{n+1}}{\overline{k_{n+1}}}, \\
z_{n+1}=z\left(o_{n+1}\right)-\frac{\cos \theta_{n+1}}{\overline{k_{n+1}}} .
\end{array}\right.
$$

According to the above mathematical relationship, when the coordinates and curvature of the starting point are known, the coordinate value of any point on the curve can be deduced to realize the fitting of the entire curve. At the same time, because the error of each point is cumulative, when the number of discrete points is large, the error superposition is large. Later in this paper, the data value obtained by finite element simulation will be modified to this recursive way to reduce the error.

\section{Cooperative Finite Element Analysis of Geogrid and Surrounding Rock Deformation}

In practical engineering, the supporting material and surrounding rock are often analyzed as a whole. Since the smart geogrid material has not yet been practically applied in engineering, in order to analyze the feasibility of its sensing principle in tunnel engineering, select parameters according 
to relevant engineering cases [23-25] and use PLAXIS 3D finite element software to analyze the geogrid and surrounding rock deformation synergy mechanism under two different construction processes (the full-face excavation method and two-step excavation method) and two different support forms (lining + steel-plastic geogrid and lining + steel-plastic geogrid + anchor rod), seek the optimal layout of the smart geogrid, and optimize its discrete curvature sensing principle.

3.1. Calculation Model and Parameters. The rock mass is simulated by the Mohr-Coulomb ideal plastic model, the shotcrete is simulated by virtual slab element, and the geogrid is QLGS80-80 steel-plastic geogrid [26]. $\mathrm{EA}=3 \times 10^{3} \mathrm{KN} / \mathrm{m}, f_{\mathrm{t}}=80 \mathrm{KN} / \mathrm{m}$, can be simulated with PLAXIS 3D own grille unit. In order to better represent the interaction between anchor and rock mass, the embedded beam element is used to simulate the anchor, and the parameters of each supporting structure are shown in Tables 1 and 2 .

In the process of simulated excavation, it is considered that the materials are all ideal elastoplastic bodies, and the plane strain problem is used to simulate, and the creep effect of rock is not considered. Considering the model boundary influence, the model has a size of $40 \mathrm{~m} \times 40 \mathrm{~m} \times 70 \mathrm{~m}$, in the $x, y$, and $z$ directions. The left and right boundaries apply a constraint load, and the upper boundary is free. The model is shown in Figure 3.

Considering the influence of different supporting structures on geogrid strain network and deformation reconstruction, the supporting structure model is shown in Figure 4.

3.2. Preconstruction Treatment. Because the tunnel construction was excavated by sections, each construction step can be simulated by the activation and freezing of the unit in the PLAXIS 3D software. The model width boundary is $40 \mathrm{~m}$. For the convenience of calculation, $20 \mathrm{~m}$ is selected as the excavation boundary, the tunnel footage is excavated $2 \mathrm{~m}$ for each construction step, and the supporting construction distance is $2 \mathrm{~m}$ from the excavation section. In the PLAXIS 3D software, under the tunnel editor track sub-block, the construction footage is simulated by setting the slice length to $2 \mathrm{~m}$. Under the sequencing sub-block, the TBM excavation method is selected, and the freezing of the soil and the activation of the lining, anchors, and geogrid are simulated in each construction step to simulate each construction step, thereby realizing the simulation of the entire tunnel construction excavation. To satisfy the number of discrete point curvature values, it is assumed that the geogrid element has been activated before excavation.

3.3. Simulation Results and Analysis. The structural node elements are selected in the PLAXIS 3D postprocessing program output to view the numerical simulation
TABLE 1: Parameters of surrounding rocks and lining materials.

\begin{tabular}{lcccccc}
\hline & $d$ & $\gamma\left(\mathrm{kN} \mathrm{m}^{-3}\right)$ & $E$ & & $\varphi$ \\
$(\mathrm{GPa})$ & $\nu$ & $\begin{array}{c}\varphi \\
\left({ }^{\circ}\right)\end{array}$ & $C(\mathrm{MPa})$ \\
\hline Surrounding rocks & 50 & 22 & 1 & 0.34 & 37 & 0.2 \\
Shotcrete & 0.2 & 22 & 30 & 0.25 & & \\
\hline
\end{tabular}

calculation results. The deformation value of the surrounding rock at the vault and the strain value of the geogrid is selected (the geogrid strain value is obtained by the grid axial force N1 and the tensile stiffness EA), and the relationship between the two is established according to the theory in Section 2, in order to analyze the response of the smart geogrid to the deformation of surrounding rock under different construction techniques and supporting forms.

3.3.1. Effects of Construction Techniques. In order to facilitate the calculation and curve reconstruction, it is considered that the displacement at the tunnel entrance is known and tends to be stable, and the geogrid shaft force diagram at the last construction stage is extracted, as shown in Figure 5. It can be seen that the geogrid shaft force at the vault under the two construction techniques changes uniformly, and there is no bad element node, and the axial force (strain) at the tunnel entrance and nearby is more consistent, which can be used as the starting point of curve reconstruction.

To prevent the influence of boundary effect, $1 \mathrm{~m}$ is selected from $5-35 \mathrm{~m}$ as an interval and the geogrid strain value is selected for deformation reconstruction according to the principle of discrete curvature reconstruction curve shown in Section 2.2, and the settlement curve and deformation reconstruction curve of the vault are shown in Figure 6.

According to Wang [18], the error of discrete curvature reconstruction increases linearly. The curve is divided into2 two sections: the curve before excavation face and the curve after excavation face, and the error is corrected according to the settlement of the vault of the end points of the two curves (the settlement value of the vault at $35 \mathrm{~m}$ is taken as $0 \mathrm{~mm}$ ). The corrected curve is shown above. It can be seen that the reconstruction degree of the curve before excavation face is relatively high after correction, while the curve after excavation face still has a certain distance. Especially near the excavation section, there is a large error in curve reconstruction due to the spatial effect of surrounding rock deformation. Under the construction of the two-step method, because the displacement of surrounding rock is relatively stable, the reconstruction degree of deformation curve is relatively high for the full-face method.

3.3.2. Effects of Support Forms. In order to compare the influence of support form on deformation reconstruction, four working conditions of shotcrete thickness $d=15 \mathrm{~cm}$ and $30 \mathrm{~m}$ and anchor length $L=2 \mathrm{~m}$ and $4 \mathrm{~m}$ were selected to simulate by controlling variables. Choose $1 \mathrm{~m}$ from $5-35 \mathrm{~m}$ 
TABLE 2: Material parameters of anchor.

\begin{tabular}{ccccccc}
\hline $\mathrm{d}(\mathrm{m})$ & $\gamma\left(\mathrm{kN} \mathrm{m}^{-3}\right)$ & $\mathrm{E}(\mathrm{GPa})$ & Length $(\mathrm{m})$ & Longitudinal spacing $(\mathrm{m})$ & Radial spacing $(\mathrm{m})$ & Support lag interval $(\mathrm{m})$ \\
\hline 0.022 & 8 & 210 & 2 & 1.5 & 2.0 & 2.0 \\
\hline
\end{tabular}

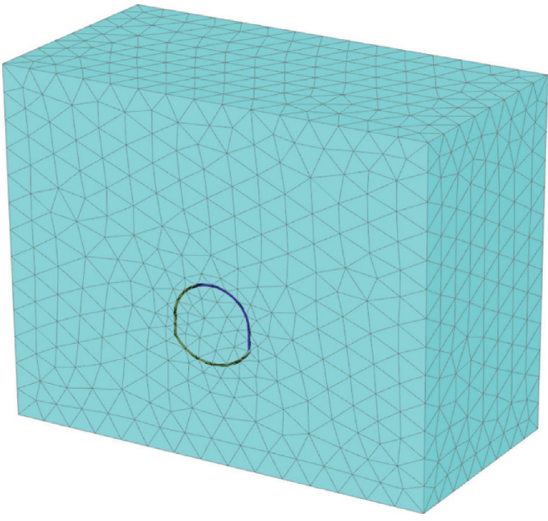

(a)

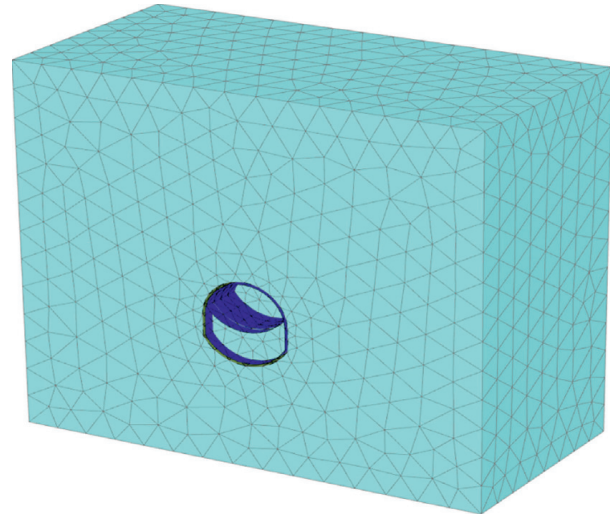

(b)

FIgURe 3: Excavation and dimensional boundary model: (a) full-face method; (b) two-step method.

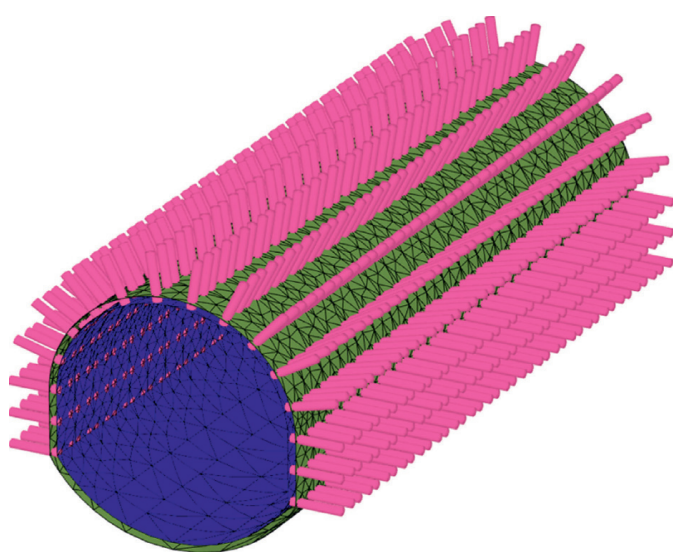

(a)

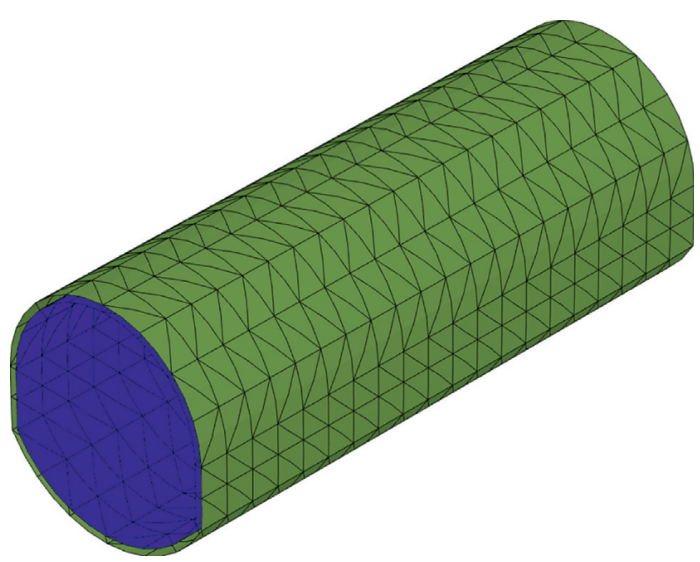

(b)

FIGURE 4: Support structure model: (a) lining + geogrid + anchor; (b) lining + geogrid.

as an interval to select the geogrid strain value for deformation reconstruction. The curve of vault settlement and deformation reconstruction curve are shown in Figure 7.

From Figure 7, it can be seen that the thickness of lining has a great influence on deformation reconstruction. When the lining is taken to $30 \mathrm{~cm}$, most of the surrounding rock pressure is borne by the lining, making the geogrid strain greatly reduced under the influence of excavation disturbance and surrounding rock, and the deformation reconstruction curve and modified curve are close to the original settlement curve, which provides the basis for the application of smart geogrid in practical engineering, so that the optimum lining thickness under smart geogrid support is possible. In addition, due to the strong integrity of geogrid material and concrete lining, a rigid connection is formed between the bolt and geogrid, leading to a small strain of the geogrid at the rigid connection of bolt. It cannot truly reflect the impact of the settlement deformation of the vault on the strain of the geogrid, resulting in a larger error in deformation reconstruction under the action of bolt support than other support methods. It is suggested to select the appropriate discrete point curvature under the form of anchor bolt support for deformation reconstruction or performing field experimental research.

\subsection{Error Optimization of Deformation Reconstruction.} Figures 6 and 7 show that the linear error correction theory of reference [18] is well reflected in the curve before excavation face, and the curve after deformation reconstruction 


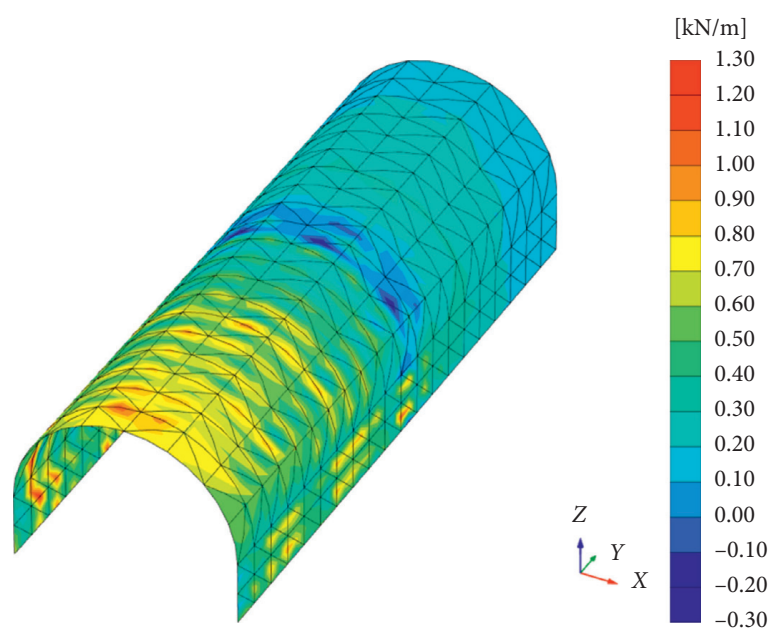

(a)

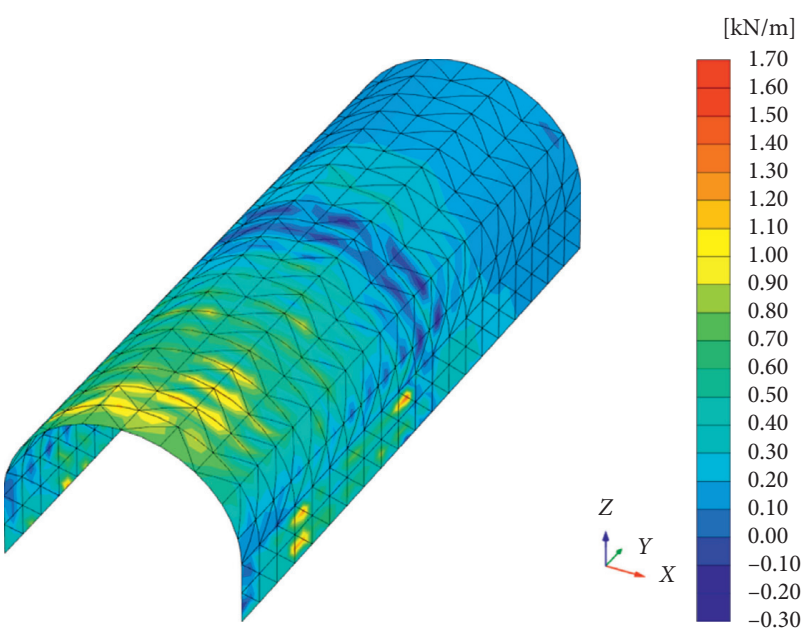

(b)

Figure 5: Geogrid axial force cloud diagram: (a) full-face excavation method; (b) two-step excavation method.

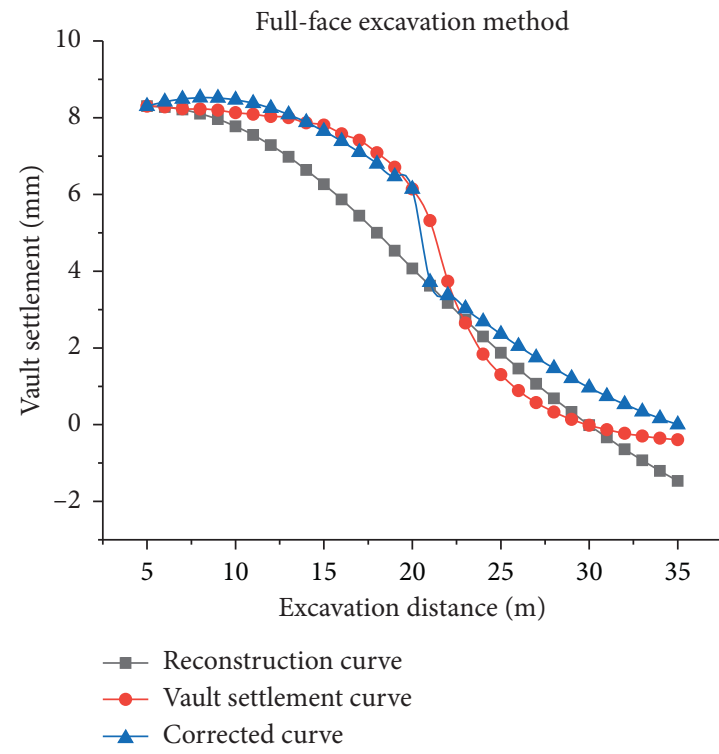

(a)

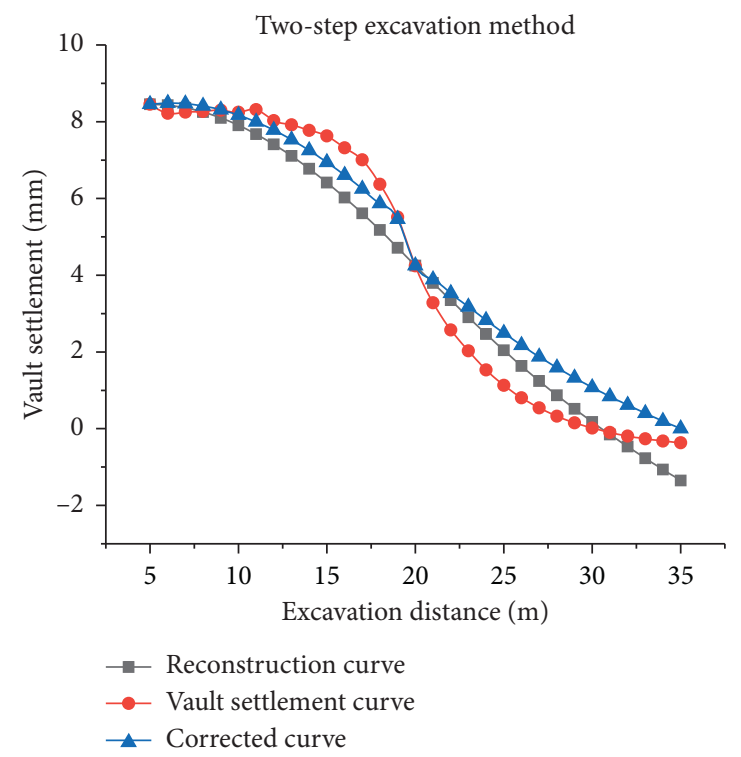

(b)

Figure 6: Displacement settlement curve.

is close to the original settlement curve, but there is a large error near the excavation face and after the excavation face. It remains to be discussed whether this error correction is applicable in the face of complex and diverse practical tunnel engineering. The principle of curve reconstruction is to select the discrete curvature of the microarc; the arc itself is a quadratic power polynomial, and the error of the horizontal coordinate is analyzed under the six working conditions of Figures 6 and 7. The results are as follows.

It can be seen from Figure 8 that the working condition of $d=30 \mathrm{~cm}$ is the slowest error superposition in the six working conditions, which proves the conclusion of
Section 3.3.2. In addition, the setting of anchor rod affects the local strain value of geogrid, which leads to the error superposition acceleration rate of its reconstruction curve becoming the fastest rate in the six working conditions. The error superposition of $0-4 \mathrm{~m}$ sections of the curve before excavation face is almost 0 , so it is feasible to use the theory of reference [18] to make linear correction of curve error before excavation face. In the curve after excavation face, it can be seen that the error acceleration rate is faster and the difference between the six working conditions is large. After fitting, it is found that the error of the abscissa of the curve increases by a second power 


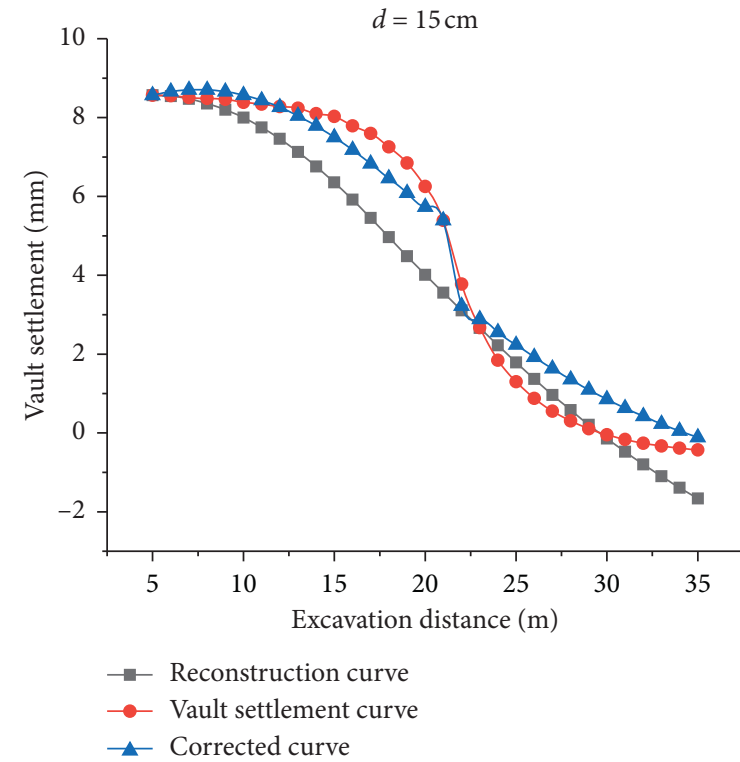

(a)

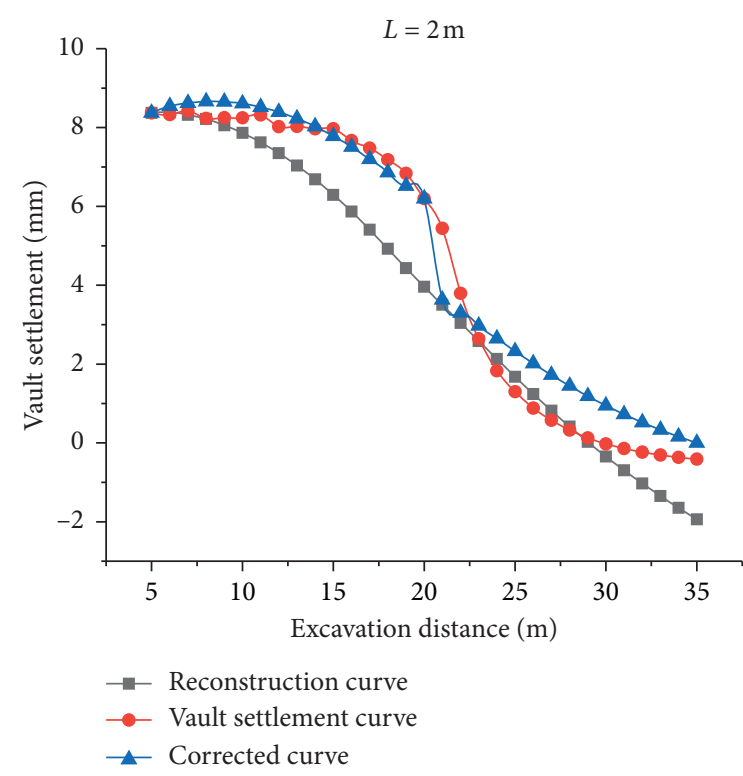

(c)

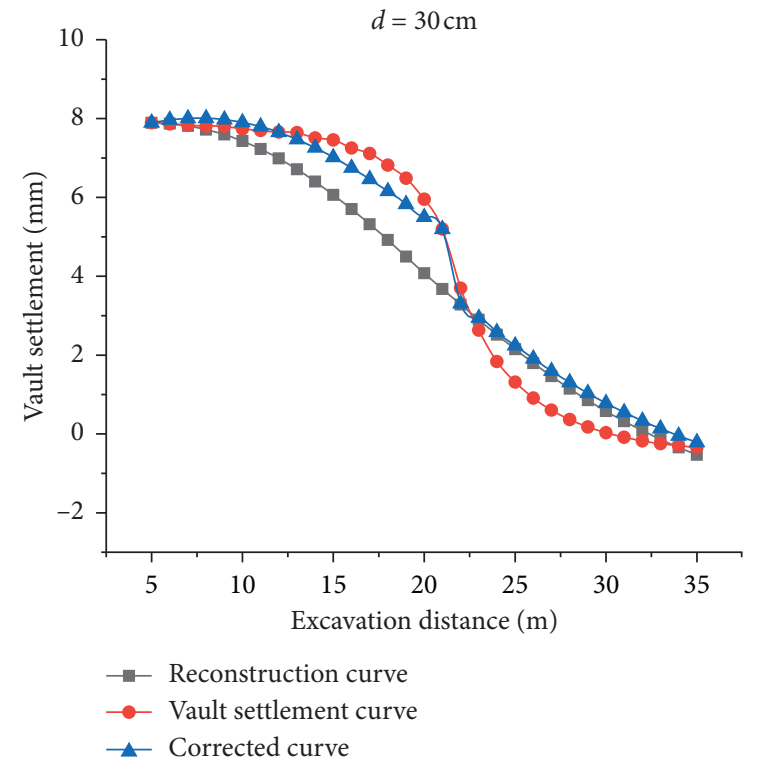

(b)

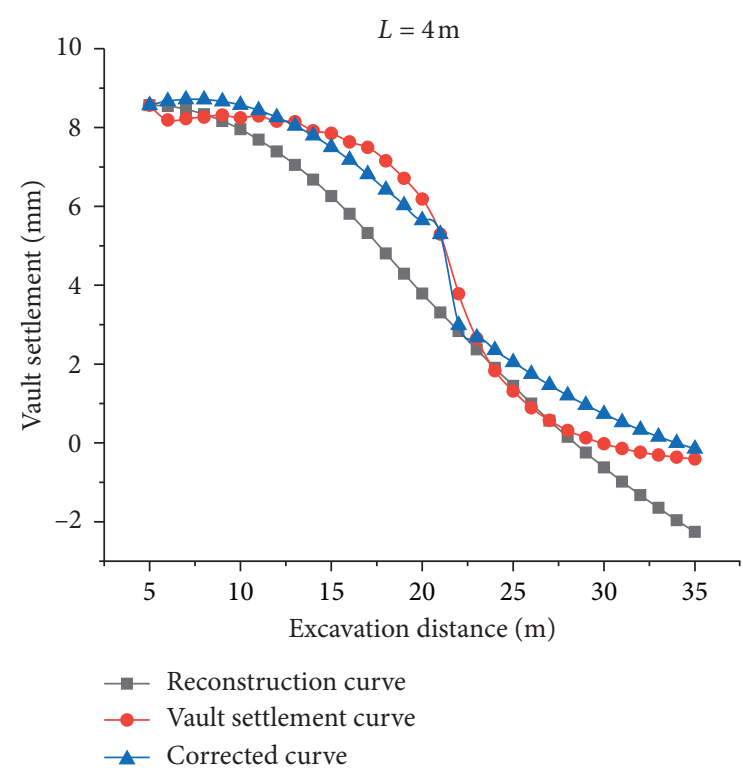

(d)

Figure 7: Deformation reconstruction under the influence of support form.

after excavation face. It is assumed that the error superposition of ordinate coordinates increases to the second power as well as that of abscissa; suppose that the starting coordinates of the ordinate error fitting curve are $p_{1}\left(x_{1}, y_{1}\right)$ and the end point coordinates are $p_{n}\left(x_{n}, y_{n}\right)$. Linear error correction is adopted for the curve before excavation face; for the curve after excavation face, the error correction function is set as follows:

$$
y=a x^{2} .
$$

Bring the starting point coordinates $p_{1}$ and ending point coordinates $p_{n}$ into the formula and get the value of $a$ :

$$
a=\frac{y_{n}-y_{1}}{x_{n}^{2}-x_{1}^{2}} .
$$

After correction, the end point coordinates are $p_{n}^{\prime}\left(x_{n}^{\prime}, y_{n}^{\prime}\right)$, and the error correction curve is as follows:

$$
y^{\prime}=y-\frac{y_{n}-y_{1}}{x_{n}^{2}-x_{1}^{2}} \cdot x^{2}
$$

Correct Section 3.3.1 according to this error correction curve. The curve of the linear superposition error correction theory in reference [18] is corrected curve I, and the error correction curve in this paper is corrected curve II. The modified deformation curve is as follows. 


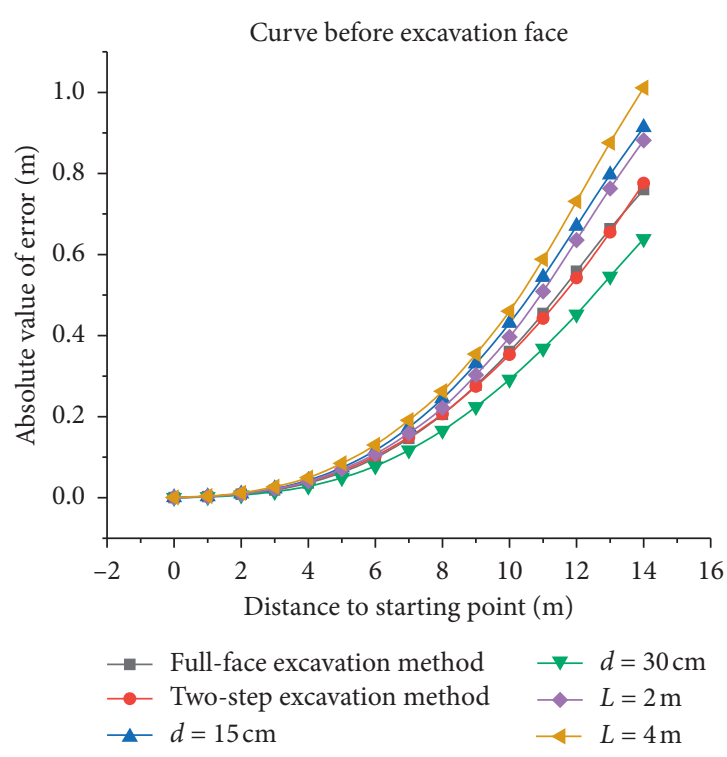

(a)

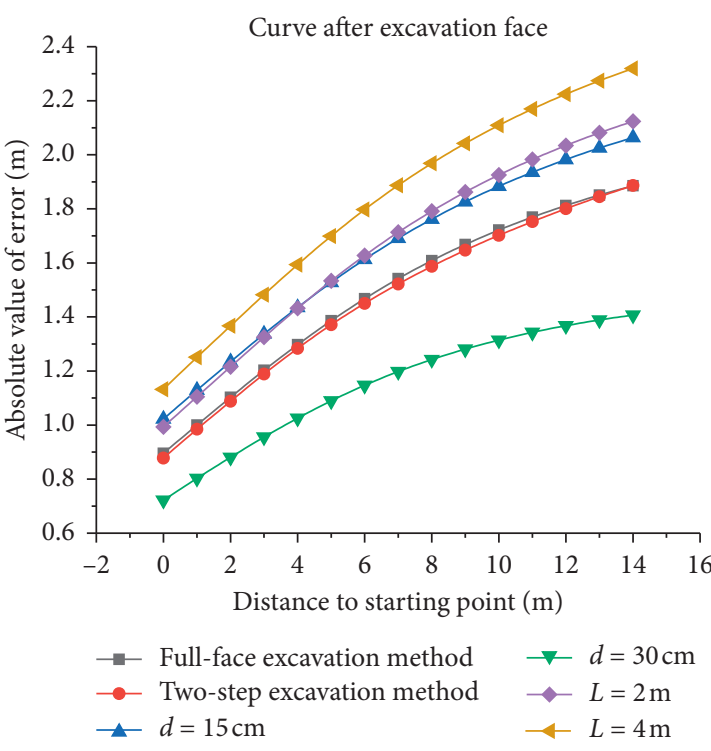

(b)

FIGURE 8: Error fitting of horizontal coordinates.

It can be seen from Figure 9 that the second power error correction curve and the original settlement curve have a higher degree of fit, especially on the curve after the excavation face. Within the distance from the opening to the excavation face, the settlement displacement is less disturbed by excavation due to the influence of support implementation. The original modified theory can meet the user requirements. The surrounding rock near the excavation face and the rear part is greatly affected by the excavation disturbance, and the theory of second power correction is required. Assuming that $x=0$ is the excavation face and $x<0$ is behind the excavation face, $x>0$ is the front of the excavation face; according to the above summary, the correction curve of smart geogrid deformation reconstruction applicable to actual tunnel engineering is as follows:

$$
\left\{\begin{array}{l}
y^{\prime}=y-\frac{y_{n}-y_{1}}{x_{n}-x_{1}} \cdot x(x>0), \\
y^{\prime}=y-\frac{y_{n}-y_{1}}{x_{n}^{2}-x_{1}^{2}} \cdot x^{2}(x<0) .
\end{array}\right.
$$

3.5. Optimal Laying of Smart Geogrid. In addition to the error superposition of the curve reconstruction principle itself, the location of the FBG smart geogrid measurement points is also an important factor that affects the calculation results and the settlement displacement fitting. The principle is to use the geogrid strain value at the measuring point to reconstruct the deformation, so the geogrid strain value discrete points which can correctly reflect the surrounding rock settlement deformation and which are not affected by other structural components can be selected as the optimal layout of smart geogrid. The geogrid axial force N1 of vault under $x$-axis of local coordinate axis in six working conditions is extracted as follows.
As can be seen from Figure 10, due to the support form and excavation disturbance, there is a certain fluctuation in the axial force of the geogrid; especially in the case of anchor bolt support, the axial force fluctuation reaches the maximum value. Since the simulation excavation footage is set to advance by $2 \mathrm{~m}$ in each construction stage, in the form of non-anchor bolt support, the axial force value of the end geogrid of each $2 \mathrm{~m}$ footage changes greatly compared with the surrounding parts, and when the lining thickness is set as $30 \mathrm{~cm}$, the change is small. In the form of anchor bolt support, due to the rigid connection between the anchor rod and the concrete lining, the axial force of the anchoring end geogrid is small, while the geogrid axial force at the middle span of the adjacent anchor bolt is larger, resulting in the axial force fluctuation as shown in Figure 10. In the case of axial force fluctuations, selecting different measurement points for deformation reconstruction will produce unequal errors.

According to the above summary, in the form of nonanchor bolt support, the end point (selection point I) and intermediate point (selection point II) of every $2 \mathrm{~m}$ footage are selected, respectively. In the form of anchor bolt support, the middle point of adjacent anchor bolt span (selection point I) and anchor end point (selection point II) are selected, respectively. According to the error correction theory of formula (12), the deformation reconstruction is carried out. Taking two working conditions of $d=15 \mathrm{~cm}$ and $L=2 \mathrm{~m}$ as examples, the results are as follows.

According to Figure 11, choosing different FBG measuring point layout methods will also lead to large errors, and the fluctuation of axial force will also affect the fitting degree of curves after reconstruction, especially in the form of anchor bolt support. It can be seen from the axial force diagram of the geogrid under the working condition of $L=2 \mathrm{~m}$ in Figure 10 that the strain of the geogrid is generally small in the case of selecting point II, and there are even 


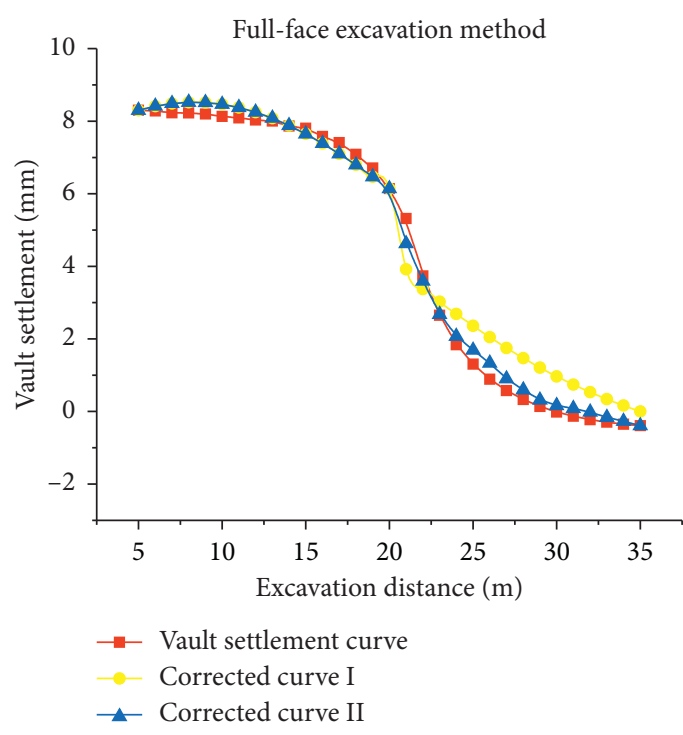

(a)

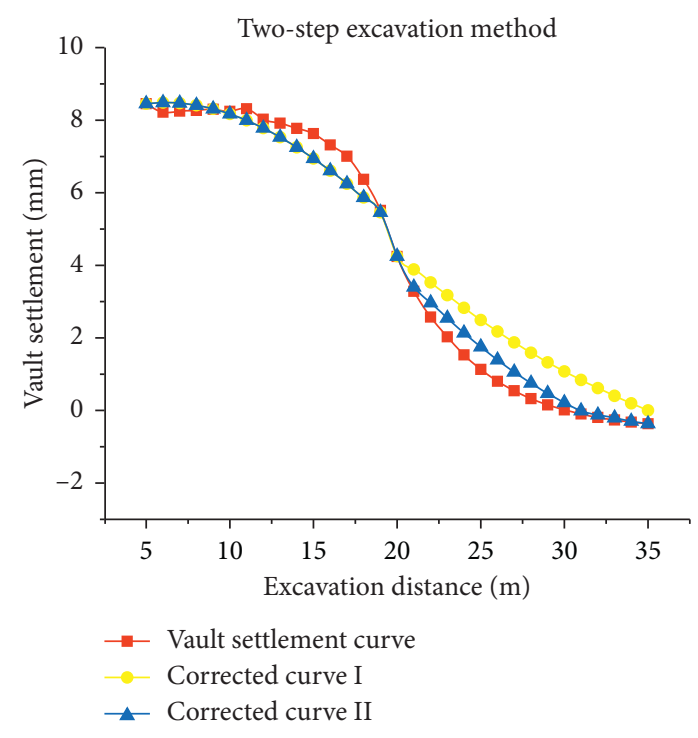

(b)

FIgURE 9: Error correction curve.

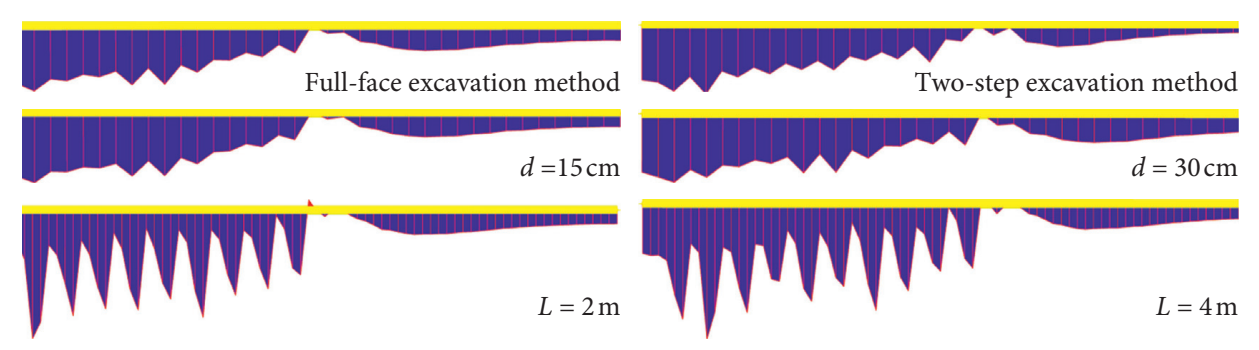

FIgURE 10: Geogrid axial force diagram.

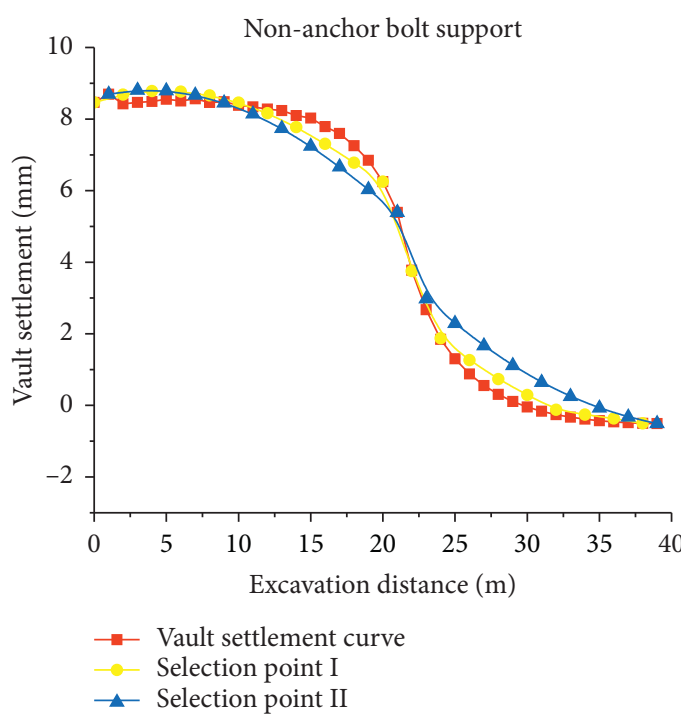

(a)

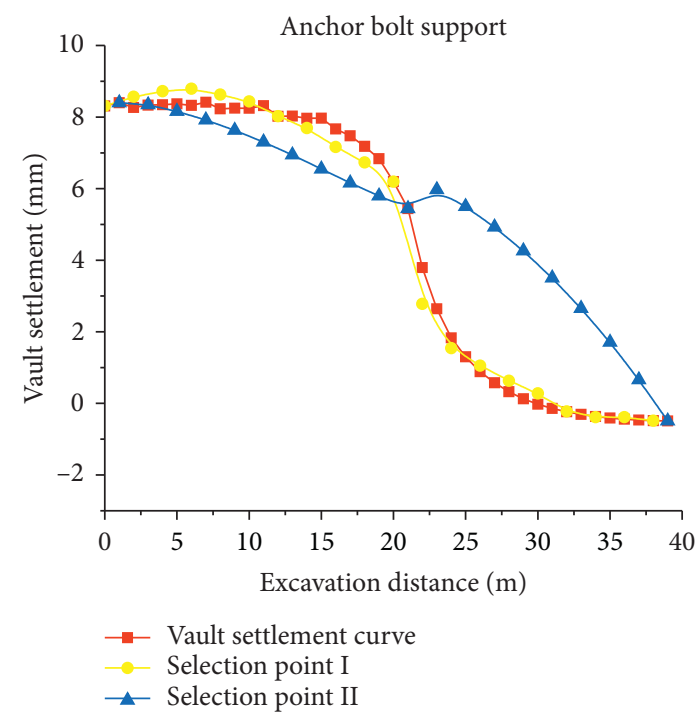

(b)

FIGURE 11: Deformation reconstruction under different selection points. 
cases of 0 or negative values, making the curvature radius of adjacent discrete points become large, resulting in the arc between the two discrete points approximate to a straight line, corresponding to the curves before and after excavation face at the case of selection point II in Figure 11.

The error of deformation reconstruction by selecting point I is relatively small, that is, in the form of non-anchor bolt support, the end of each excavation footage is selected as the location of FBG smart geogrid measuring points; in the form of anchor bolt support, the measuring points are selected at intervals between the midpoints of adjacent anchors. This arrangement can reduce unnecessary errors in the selection of measuring points and facilitate data processing and calculation.

\section{Conclusions}

PLAXIS 3D finite element software was used to analyze the feasibility of applying the principle of discrete curvature deformation reconstruction under the cooperative action of smart geogrid and tunnel surrounding rock in practical engineering. On this basis, considering the construction method and the form of support, an optimal design is proposed for its error stacking, and the optimal layout of the smart geogrid is explored. The following conclusions are drawn.

(1) When the absolute value or relative value of the settlement displacement at the starting point of the tunnel opening and the excavation surface is determined, the principle of discrete curvature reconstruction settlement curve is feasible in practical engineering, and the degree of reconstruction on the curve before excavation face is high. Concrete lining support is the smallest error superposition among all supporting forms, and the thicker the lining thickness, the smaller the reconstruction superposition error and the larger the error superposition of the anchor bolt support form; it is recommended to select the appropriate discrete point curvature for deformation reconstruction or development field experimental research.

(2) The error correction curve of the original deformation reconstruction principle is not universal. In view of the low degree of curve reconstruction after the excavation surface, the theory that the curve before the excavation face is linearly modified and the curve after the excavation face is the second power correction is adopted. It is verified by the finite element simulation of the actual working conditions that the quadratic power correction theory makes the reconstructed curve have a higher fitting degree.

(3) Under different construction methods and support forms, the geogrid axial force fluctuates to a certain extent, and the setting of anchor rod affects the local strain value of geogrid, resulting in the maximum fluctuation of geogrid axial force; in the form of anchor bolt support, select the midspan points of adjacent bolts to arrange the measuring points at intervals. In the form of non-anchor bolt support, choose the end of each excavation footage as the location of the FBG smart geogrid measurement point. The layout method can reduce unnecessary errors caused by the selection of measuring points.

\section{Data Availability}

The raw/processed data required to reproduce these findings cannot be shared at this time as the data also form part of an ongoing study.

\section{Conflicts of Interest}

The authors declare that they have no conflicts of interest.

\section{Acknowledgments}

This study was supported by the National Natural Science Fund Project (grant no. 41877230) and Open Fund of State Key Laboratory of Mechanical Behavior and System Safety of Traffic Engineering Structures (KF020-23).

\section{References}

[1] C. Tian, Z. Wang, Q. Sui et al., "Design and optimization of FBG implantable flexible morphological sensor to realize the intellisense for displacement," Sensors, vol. 18, no. 7, p. 2342, 2018.

[2] R. J. Roesthuis, M. Kemp, and J. J. Misra, "Three-Dimensional needle shape reconstruction using an array of fiber bragg grating sensors," IEEE/ASME Transactions on Mechatronics, vol. 19, no. 4, pp. 1115-1126, 2014.

[3] H.-H. Zhu, J.-H. Yin, L. Zhang, W. Jin, and J.-H. Dong, "Monitoring internal displacements of a model dam using FBG sensing bars," Advances in Structural Engineering, vol. 13, no. 2, pp. 249-261, 2010.

[4] M. F. Mendez, N. L. Adams, and K. S. Lewandowski, "Neurobehavioral changes associated with caudate lesions," Neurology, vol. 39, no. 3, p. 349, 1989.

[5] F. Tavenas, C. Mieussens, and F. Bourges, "Lateral displacements in clay foundations under embankments," $\mathrm{Ca}$ nadian Geotechnical Journal, vol. 16, no. 3, pp. 532-550, 1979.

[6] R. L. Idriss, "Monitoring of a smart bridge with embedded sensors during manufacturing, construction and service," in Proceedings of the 3rd International Workshop on Structural Health Monitoring, pp. 604-613, Stanford, CA, USA, September 2001.

[7] J. Ou and W. U. Z. Zhouzhi, "Intelligent monitoring of Heilongjiang Hulanriver bridge based on FBGS," China Civil Engineering Journal, vol. 37, no. 1, pp. 45-50, 2004.

[8] D. Inaudi, "Application of optical fiber sensor in civil structural monitoring," in Proceedings of SPIE, Fiber Grating Sensors and their Application, pp. 1-10, Calcutta, India, February 2001.

[9] X. X. Guo, B. Wang, Z. Y. Wang, W. Yu, Z. Ma, and T. Yang, "Application of the microclamped fiber bragg grating (FBG) sensor in rock bolt support quality monitoring," Advances in Civil Engineering, vol. 2020, Article ID 3419835, 10 pages, 2020.

[10] H. Y. Peng, B. L. Chen, P. Dong, S. Chen, Y. Liao, and Q. Guo, "Application of FBG sensing technology to internal 
deformation monitoring of landslide," Advances in Civil Engineering, vol. 2020, Article ID 1328945, 10 pages, 2020.

[11] C. Zhu, K. Zhang, H. Cai et al., "Combined application of optical fibers and CRLD bolts to monitor deformation of a pitin-pit foundation," Advances in Civil Engineering, vol. 2019, Article ID 2572034, 16 pages, 2019.

[12] C. T. Gnanendran and A. P. S. Selvadurai, "Strain measurement and interpretation of stabilising force in geogrid reinforcement," Geotextiles and Geomembranes, vol. 19, no. 3, pp. 177-194, 2001.

[13] R. S. Bhamber, T. Allsop, G. Lloyd, D. J. Webb, and J. D. AniaCastanon, "Arbitrary real-time three-dimensional corporal object sensing and reconstruction scheme," Optics Letters, vol. 37, no. 17, pp. 3549-3551, 2012.

[14] L. Xu, J. Ge, J. H. Patel, and M. P. Fok, "Dual-layer orthogonal fiber Bragg grating mesh based soft sensor for 3-dimensional shape sensing," Optics Express, vol. 25, no. 20, pp. 24727-24734, 2017.

[15] H. Yazdani, K. Hatami, and B. P. Grady, "Sensor-enabled geogrids for performance monitoring of reinforced soil structures," Journal of Testing and Evaluation, vol. 44, no. 1, pp. 391-401, 2016.

[16] H. Yazdani and K. Hatami, "Sensor-enabled geogrids for stabilization and performance monitoring of earth structures: state of development," International Journal of Geosynthetics \& Ground Engineering, vol. 2, no. 4, 37 pages, 2016.

[17] J. Wang, S. C. Li, B. Shi et al., "Trifarious FBG sensor strain transfer characteristics and its application to tunnel excavation model test," Journal of Engineering Geology, vol. 21, no. 2, pp. 182-189, 2013.

[18] Z. F. Wang, "Study on fiber bragg grating sensing theory and key technology for bridges and tunnels engineering safety monitoring," Dissertation, Shandong University, Jinan, China, 2014.

[19] T. Wei, "Study on convergence form perception principle of roadway surrounding rock based on FBG intelligent grille," Dissertation, China Mining University, Xuzhou, China, 2019.

[20] J. Q. Wu, D. Y. Yang, L. Y. Shen et al., "Research on the curvefitting method based on curvature data," Journal of Applied Sciences, vol. 21, no. 3, pp. 258-262, 2003.

[21] X. J. Zhu, H. S. Zhang, C. N. Xie et al., "Analysis of curve surface fitting algorithm based on curvatures for space sailboard structure," Journal of System Simulation, vol. 19, no. 11, pp. 2496-2499, 2007.

[22] J. C. Yi, "Shape perception and active monitoring for smart structure using fbg sensor array," Dissertation, Shanghai University, Shanghai, China, 2014.

[23] B. Deng, H. G. Rao, W. P. Liao et al., "Optimization of the soft rock tunnel support structure," Journal of Railway Science and Engineering, vol. 14, no. 10, pp. 2203-2213, 2017.

[24] J. Huang, Q. G. Liang, L. L. Yue et al., "Numerical simulation analysis of high ground stress soft rock tunnel deformation subject to different construction methods," Railway Standard Design, vol. 5, pp. 116-119, 2018.

[25] J. W. Chang, "Longitudinal analysis and relate research of tunnel support based on convergence-confinement method," Dissertation, Beijing Jiaotong University, Beijing, China, 2019.

[26] Y. J. Liu, Y. P. Song, and J. Y. Wang, "Analysis of steel plastic network applied in tunnel supporting," Technology of Highway and Transport, vol. 1, pp. 134-137, 2007. 